\title{
openheart Fractional flow reserve in acute coronary syndrome: a meta-analysis and systematic review
}

\author{
Kevin P Liou, ${ }^{1,2,3}$ Sze-Yuan M Ooi, ${ }^{2,3}$ Stephen $\mathrm{P}$ Hoole, ${ }^{1}$ Nick E J West ${ }^{1}$
}

\begin{abstract}
- Additional material is published online only. To view please visit the journal online (http://dx.doi.org/10.1136/ openhrt-2018-000934).
\end{abstract}

To cite: Liou KP, Ooi S-YM, Hoole SP, et al. Fractional flow reserve in acute coronary syndrome: a meta-analysis and systematic review. Open Heart 2019;6:e000934. doi:10.1136/ openhrt-2018-000934

Received 14 September 2018 Revised 5 October 2018 Accepted 12 November 2018

D Check for updates

(C) Author(s) (or their employer(s)) 2019. Re-use permitted under CC BY-NC. No commercial re-use. See rights and permissions. Published by BMJ.

${ }^{1}$ Department of Interventional Cardiology, Royal Papworth Hospital, Cambridge, UK ${ }^{2}$ Eastern Heart Clinic, Prince of Wales Hospital, Sydney, Australia ${ }^{3}$ Prince of Wales Clinical School, University of New South Wales, Sydney, Australia

Correspondence to Dr Kevin P Liou; K_liou@ hotmail.com

\begin{abstract}
Background The utility of fractional flow reserve (FFR) to guide revascularisation in the management of acute coronary syndrome (ACS) remains unclear.

Objective This study aims to compare the clinical outcomes of patients following FFR-guided revascularisation for either ACS or stable angina (SA) and in particular focuses on the outcome of those with deferred revascularisation after FFR.
\end{abstract}

Methods A meta-analysis of existing literature was performed. Outcomes including the rate of major adverse cardiovascular events (MACE), recurrent myocardial infarction (MI), mortality and unplanned revascularisation were analysed.

Results A review of 937 records yielded 9 studies comparing 5457 patients, which were included in the analyses. Patients with ACS had a higher rate of recurrent MI (OR 1.81, $\mathrm{p}=0.02)$ and a strong trend towards more MACE and all-cause mortality compared with patients with SA when treated by an FFR-guided revascularisation strategy. Deferral of invasive therapy on the basis of FFR led to a higher rate of MACE $(17.6 \%$ vs $7.3 \%$; $\mathrm{p}=0.004)$, recurrent $\mathrm{MI}(5.3 \%$ vs $1.5 \%, \mathrm{p}=0.001)$ and target vessel revascularisation ( $16.4 \%$ vs $5.6 \%$; $\mathrm{p}=0.02$ ) in patients with ACS, and a strong trend towards a higher cardiovascular mortality at follow-up when compared with patients with SA.

Conclusion The event rate in patients with ACS is much higher than SA despite following an FFR-guided revascularisation strategy. Deferring revascularisation does not appear to be as safe for ACS as it is for SA using contemporary FFR cut-offs validated in SA. Refinement of the therapeutic strategy for patients with ACS with multivessel disease is needed to redress the balance.

\section{INTRODUCTION}

The role of fractional flow reserve (FFR) in the assessment of coronary lesions of indeterminate severity is well-established for patients with stable angina (SA). An FFR-guided management approach results in better patient outcomes compared with angiography alone, ${ }^{1}$ while deferral of revascularisation for lesions above the validated physiological threshold appears safe in this setting. ${ }^{12}$

The use of FFR in the setting of acute coronary syndrome (ACS), however, remains

\section{Key questions}

What is already known about this subject?

- The role of fractional flow reserve (FFR) in the management of patients with stable angina $(\mathrm{SA})$ is well established. FFR-guided revascularisation promotes better patient outcome compared with angiography alone, while deferral of percutaneous coronary intervention (PCl) for lesions with an FFR $>0.75-0.80$ appears safe.

What does this study add?

- Deferral of revascularisation on the basis of contemporary FFR cut-off values is associated with higher event rate in patients with acute coronary syndromes (ACS) compared with patients with SA.

How might this impact on clinical practice?

- ACS is a systemic illness and therefore requires a systemic approach to therapy. FFR, as a lesion specific index, may be useful in guiding patient management in this setting, although physicians need to be aware of its limitation. Further validation of FFR in the ACS population is required to address this knowledge gap.

an area of uncertainty. While FFR-guided complete revascularisation significantly improves patient outcomes following culprit vessel primary PCI, ${ }^{3}{ }^{4}$ its use in the assessment and therefore management of culprit arteries has not been firmly established. Indeed, an FFR-guided treatment strategy has not been found to improve patient prognosis compared with angiography alone in patients with NSTEACS (online supplementary figure S2). ${ }^{15}$ Importantly, the safety of FFR-guided deferral of percutaneous coronary intervention (PCI) in patients with ACS remains unaddressed, particularly as the diagnostic threshold of 0.750 .80 has not been specifically validated in this population. Finally, the accuracy of FFR in the ACS population remains unclear. ${ }^{6-8}$

The aims of this study therefore were to determine: (1) whether an FFR-guided treatment strategy using contemporary cut-off 
values delivers similar patient outcome between those with ACS and SA and (2) if patient outcome following FFR-guided deferral of PCI differs between those with ACS and SA.

\section{METHODOLOGY}

A systematic literature search was conducted in Embase, Medline, Cochrane Database of Systematic Reviews and Cochrane Central Registers of Controlled Trials through to 15 October 2017. Reference lists of relevant studies were manually searched for additional studies. The search hedges used are listed in the supplementary material.

Studies were selected based on the following inclusion criteria: (1) prospective or retrospective studies containing information, which fulfilled the objectives of this study and (2) studies containing raw data for direct retrieval or indirect derivation for the outcomes of interests. Information was extracted from each individual study on the basis of intention-to-treat analysis. Case reports, review articles, editorials and expert opinions were excluded. When multiple manuscripts that used the same dataset were identified, only the most complete was included for assessment. Patients with SA are presumed to have stable coronary disease.

The primary outcome of interest was the major adverse cardiovascular event (MACE) rate. As MACE definitions varied between the studies, the individual elements of the composite MACE were also separately interrogated.

The data were extracted independently by two of the coauthors (KL and $\mathrm{SO}$ ) and summarised into a standardised extraction sheet. Any disagreement in data collected was resolved by consensus. Patient consent was not required for this study as it was a meta-analytic synthesis of the existing data.

\section{Statistical analysis}

Statistical analyses were performed using Review Manager V.5.3.5 and Comprehensive Meta-Analysis V.2.2.064. Between-study statistical heterogeneity was assessed using the Cochran $Q$ statistic and was quantified using the $\mathrm{I}^{2}$ method by measuring inconsistency $\left(\mathrm{I}^{2}\right.$, the percentage of total variance across studies attributable to heterogeneity rather than chance). $\mathrm{I}^{2}$ values of $25 \%, 50 \%$ and $75 \%$ correspond to low, moderate and high degrees of heterogeneity, respectively. The data were analysed by the Mantel-Haenszel methods with random effect model to account for interstudy heterogeneity. A leave-one-out analysis was performed to examine the impact of individual study on the overall results. Subgroup analyses were performed on the basis of the FFR cut-off values as well as the vessels assessed to determine their effects on patient outcomes. Sensitivity analysis was also performed to examine the incidence of revascularisation confined to the target/deferred vessels/lesions following treatment deferral. Study effect measures were expressed as ORs with their respective $95 \%$ CIs. $\mathrm{P}<0.05$ was considered statistically significant.

\section{RESULTS}

The systematic search yielded 937 records from online electronic databases. After removal of duplicates and review of contents, nine studies were included for quantitative analyses $^{5-17}$ (figure 1). Selected attributes of the studies are presented in table 1 , while the inclusion and exclusion criteria for each of the individual studies are listed in the supplementary materials (online supplementary table S1). The assessment of potential biases was made and is also displayed in the supplementary materials (online supplementary figure $\mathrm{S} 1$ ).

Overall, 5457 patients were included in the final analyses. The baseline demographics of these patients are presented in table 2, while selected lesion characteristics are presented in online supplementary table S2. Significant differences in patients' baseline attributes were noted in two studies. 1618

\section{FFR-guided management in patients with ACS and SA}

This role of FFR in the management of patients with different presentations at baseline was explored by a randomised controlled trial ${ }^{11}$ and a multinational registry ${ }^{16}$ in a total of 2361 patients. The comparisons in terms of the MACE rates, all-cause mortality, recurrent myocardial infarction (MI) and unplanned revascularisations are presented in figure 2A-D. When FFR is used to guide PCI using the standard cut-off values for revascularisation $(<0.75-0.80)$, there was a higher MACE rate in the ACS group. The rate of recurrent MI was statistically higher in those with ACS, while no difference was noted in the rate of unplanned revascularisations.

\section{FFR-guided deferral of PCI in patients with ACS}

The clinical outcomes of patients in whom PCI was deferred on the basis of the contemporary FFR cut-off values was compared between those with ACS and SA. ${ }^{9} 10$ 12-15 The results are displayed in figure 3A-D. Patients with ACS had a significantly higher rate of MACE $(17.6 \%$ vs $7.3 \%, \mathrm{p}=0.004)$ and recurrent $\mathrm{MI}(5.3 \%$ vs $1.5 \%, \mathrm{p}=0.0001)$ compared with those with $\mathrm{SA}$, while a strong trend was observed in the rate of cardiovascular mortality and unplanned revascularisations in favour of patients with SA.

Subgroup analyses restricted to studies using an FFR cut-off of 0.75 and 0.80 , respectively, and on the basis of the vessels assessed (non-culprit vs both non-culprit and culprit vessels) demonstrated no deviation from the overall trend in all outcomes with no significant differences between the subgroups (figure 3A-E).

Sensitivity analysis on the incidence of unplanned revascularisation confined to the target/deferred vessel/ lesion $^{12} 1318$ demonstrated a statistically significant rise $(16.4 \%$ vs $5.6 \%, \mathrm{p}=0.02)$ in those with ACS (online supplementary figure S3).

On the basis of the leave-one-out analysis, each individual study contributed equally and consistently to the increased incidence of MACE observed in patients with ACS (online supplementary figure S4). The overall trend 


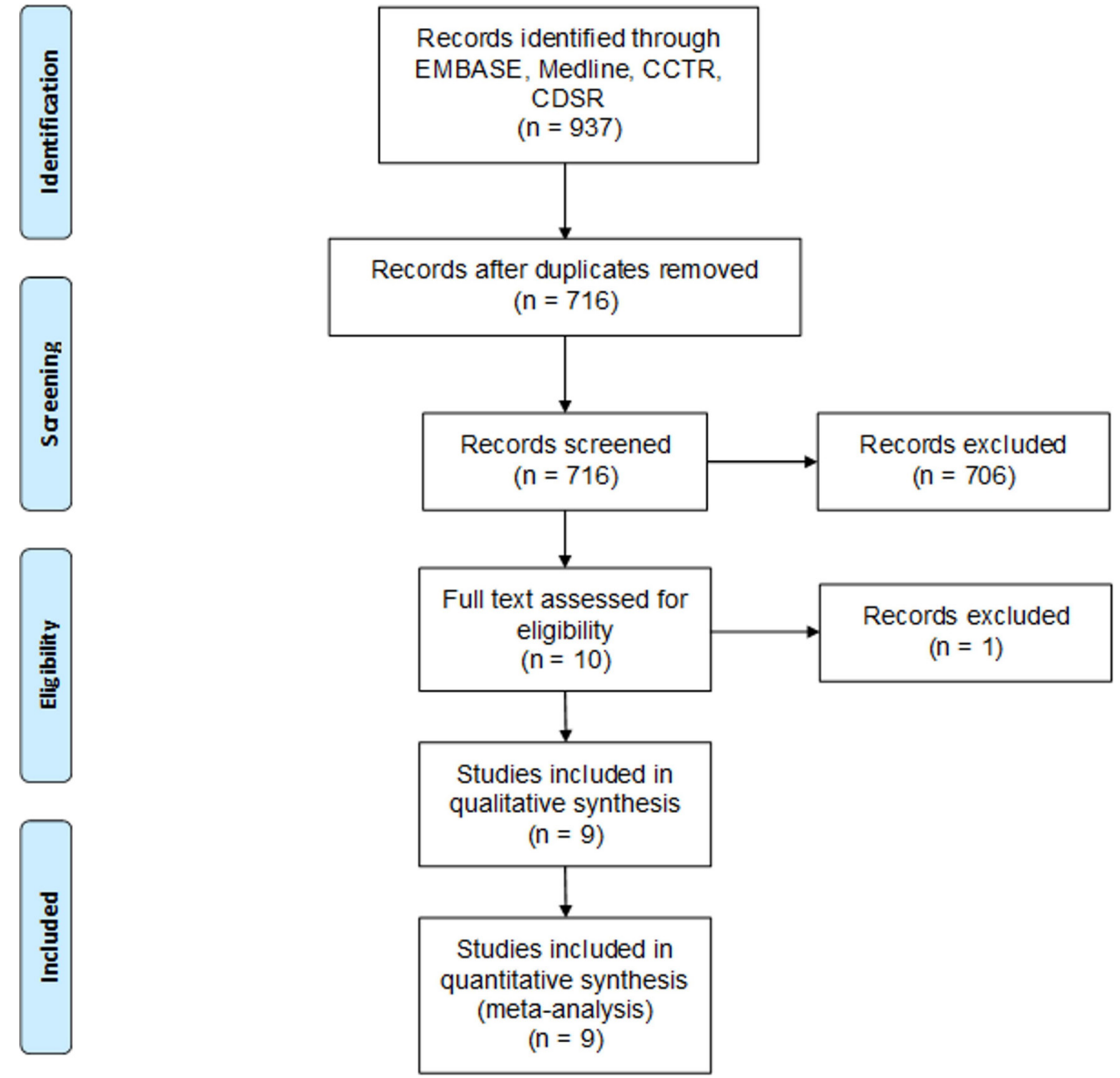

Figure 1 Preferred Reporting Items for Systematic Reviews and Meta-Analyses (PRISMA) study flow diagram.

did not vary when each study was omitted sequentially from the analysis.

\section{DISCUSSION}

The aim of this study was to examine the role of FFR in the management of patients with ACS on the basis of a meta-analysis and systematic review. When the contemporary FFR threshold values were used to guide management of patients with ACS: (1) there was a higher rate of recurrent $\mathrm{MI}$ and a strong trend towards a higher rate of MACE and all-cause mortality compared with patients with SA and (2) deferral of PCI was associated with a higher incidence of MACE, recurrent MI and target vessel revascularisation compared with those with SA, irrespective of the FFR cut-off values.

\section{Patients with ACS have higher event rates}

ACS is a systemic illness; evidence has shown that ulcerated plaques and the underlying inflammatory process are often present in territories remote from that subtended by the culprit artery, ${ }^{19} 20$ and both culprit and non-culprit lesions in ACS are more likely to be rich in lipid content than those with SA. Clinically, patients with ACS have a higher risk profile at baseline, as observed in one of the included studies. ${ }^{16}$ These fundamental differences may explain the higher recurrent MI and all-cause mortality rates observed in our ACS cohort. Indeed, studies have reported a higher rate of clinical events for each given range of FFR in those with ACS compared with SA, ${ }^{12} 18$ even when the FFR value is above the threshold for revascularisation. A systemic illness with global risk will likely require a systemic therapy, and therefore employing discrete treatment of bystander disease to mitigate this risk, even when using an FFR-guided approach, may be misguided.

\section{FFR in the management of non-culprit lesions in ACS}

The value of preventive revascularisation following culprit vessel PCI in the setting of ACS has been demonstrated previously. In the Randomized Trial of Preventive Angioplasty in Myocardial Infarction (PRAMI) study, there was a significant reduction in the primary composite endpoint of death from cardiac causes, non-fatal MI or refractory angina following angiography guided complete revascularisation. ${ }^{21}$ Similarly, there was a $10.1 \%$ absolute reduction in the primary composite endpoint of all-cause death, recurrent MI, heart failure and ischaemia driven revascularisation at 12 months in the Randomised trial of complete versus lesion-only revascularisation in patients undergoing primary PCI for STEMI and multivessel disease (CvLPRIT) study, with a strong trend in favour of complete revascularisation for all components of the composite endpoint. ${ }^{22}$ 


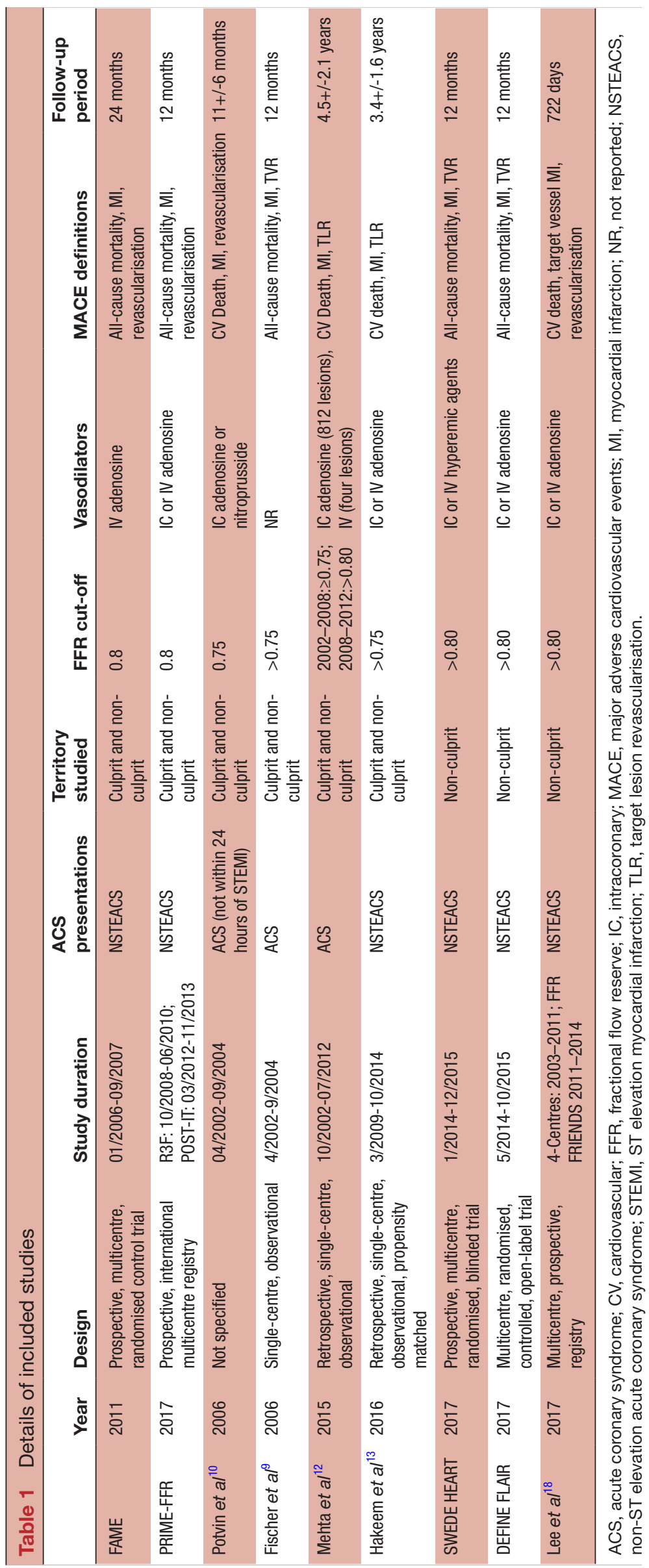




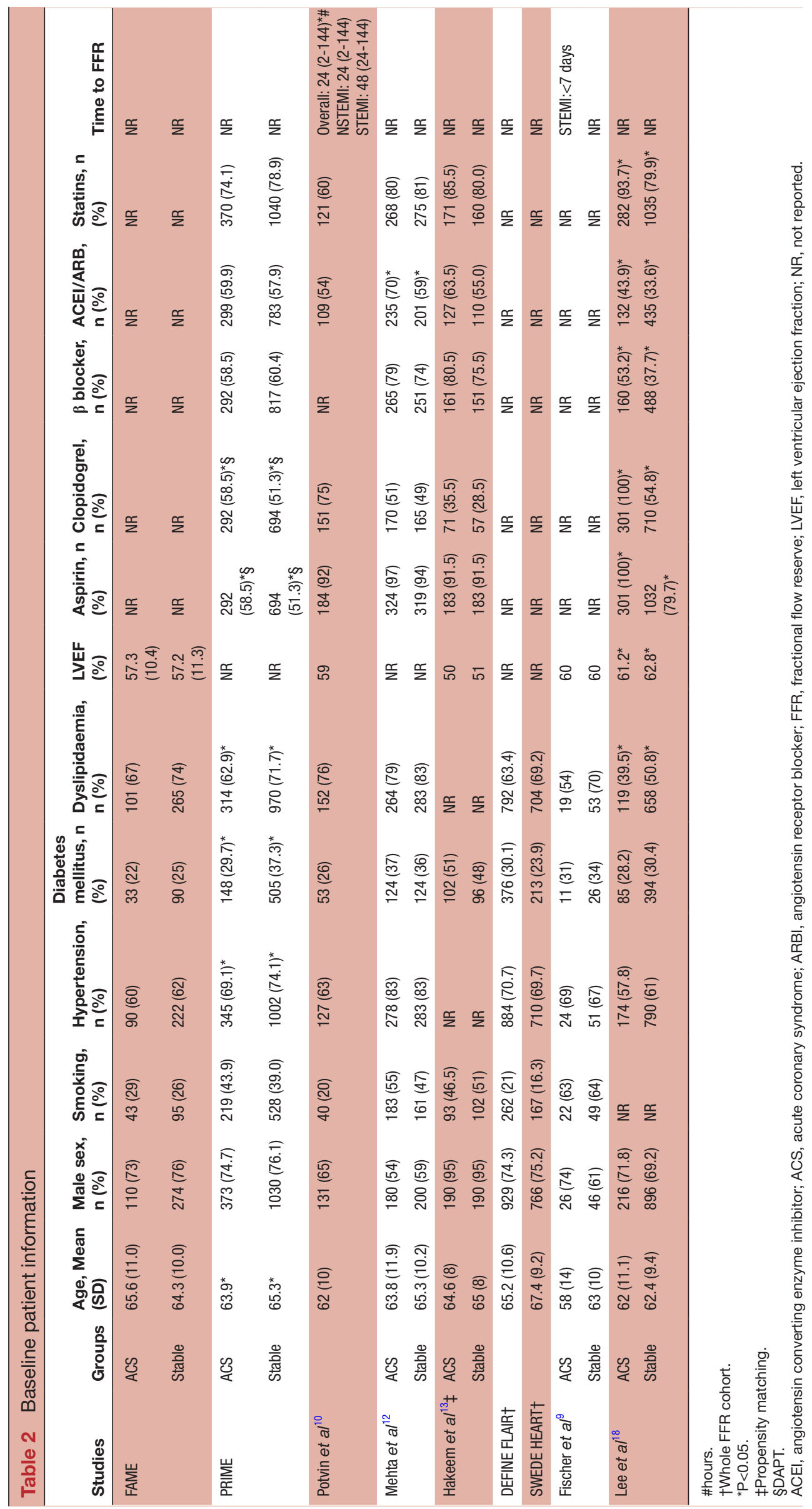


A

ACS Stable

Odds Ratio

Odds Ratio

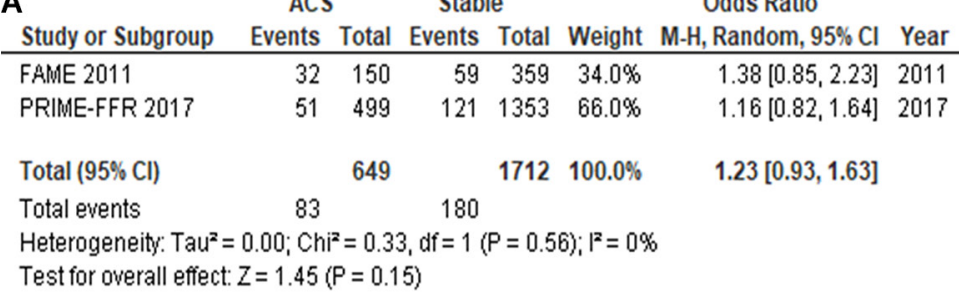

Test for overall effect: $Z=1.45(P=0.15)$

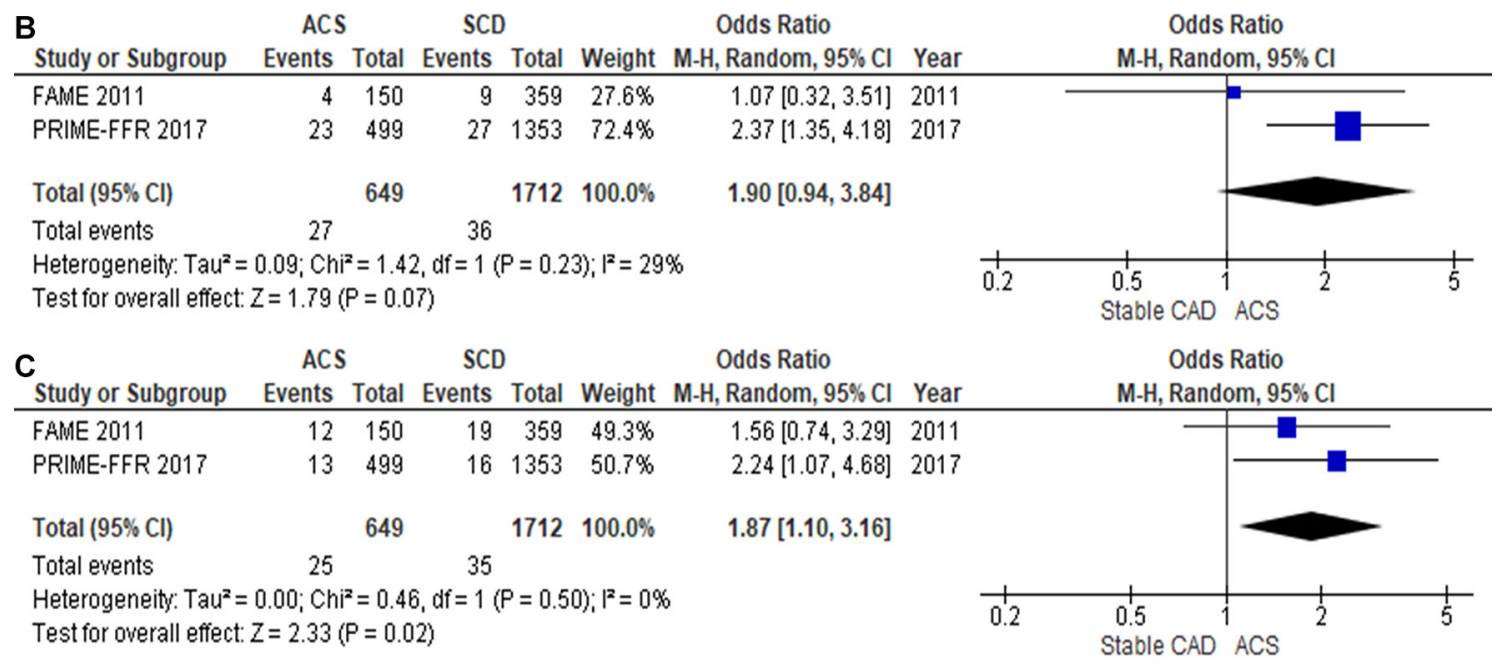

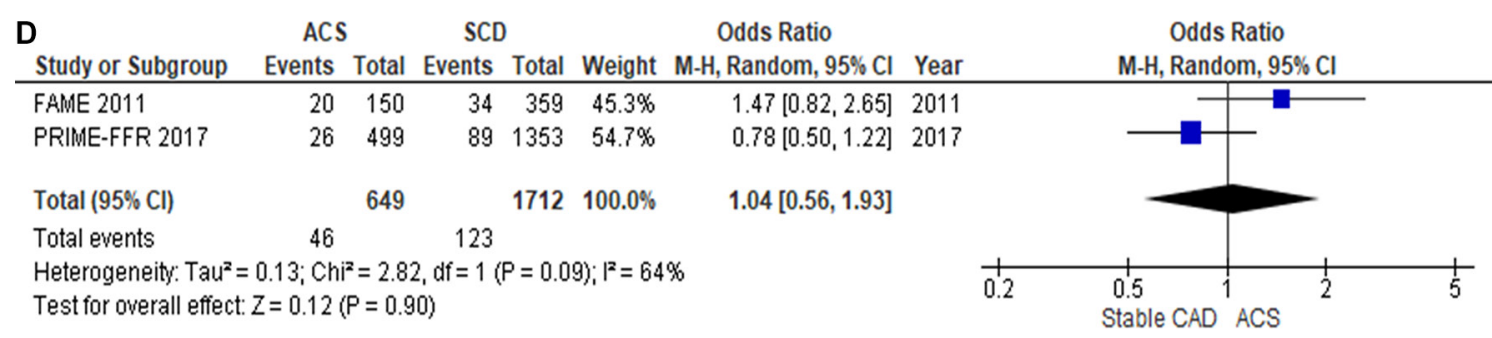

Figure 2 FFR-guided management in patients with ACS vs stable CAD. (A) Mace; (B) all-cause mortality; (C) recurrent MI; (D) unplanned revascularisation. ACS, acute coronary syndrome; FFR, fractional flow reserve; MI, myocardial infarction.

Revascularisation on the basis of angiography alone, however, risks lesion misclassification and overtreatment. This can result in an increased cost and risk of procedural complications. ${ }^{12}$ FFR, when used in conjunction with angiography, leads to a significant reduction of 'unnecessary' stent use and economic savings. Despite this, there were no detrimental effects observed in patients' clinical outcomes. Further, the stability and accuracy of FFR in non-culprit lesions have been previously demonstrated. ${ }^{723}$ Indeed, the Complete revascularisation versus treatment of the culprit lesion only in patients with ST-segment elevation myocardial infarction and multivessel disease (DANAMI-3-PRIMULTI) trial and Functional flow reserve-guided multivessel angioplasty in MI (ACUTE COMPARE) trials both demonstrated a significantly lower rate of unplanned revascularisation after FFR-guided complete revascularisation in patients who underwent primary PCI to the culprit lesions. ${ }^{34}$

The ability to permit safe deferral of invasive therapy in patients with functionally insignificant lesions therefore underpins the value of FFR. This was confirmed by the FFR-guided PCI versus medical therapy in stable coronary artery disease (FAME II) trial $^{2}$ as well as the
DANAMI-3-PRIMULTI study, where patients with FFR $>0.80$ in non-culprit vessels and had no further interventions had the same outcome as those with FFR-guided complete revascularisation following primary PCI. ${ }^{3}$

\section{FFR-guided deferral of invasive therapy}

Our meta-analysis, however, challenges the safety of deferral in patients with ACS on the basis of the FFR cut-off values established in SA; there was a significantly elevated rate of MACE, recurrent MI and culprit vessel reintervention in patients with ACS with FFR-guided deferred lesions, compared with those with SA. This was irrespective of the FFR threshold used ( 0.75 vs 0.80$)$ and vessels assessed (non-culprit versus both culprit and non-culprit vessels). This may be explained by a number of factors.

The accuracy of FFR depends on the ability to achieve maximal hyperaemia, which may be blunted in ACS due to microvascular dysfunction (MVD). MVD is common in this setting and has been attributed to a combination of neurohumoral activation, endothelial dysfunction, distal embolisation, raised left ventricular end diastolic pressure and reactive tissue oedema. ${ }^{24}$ MVD can cause 

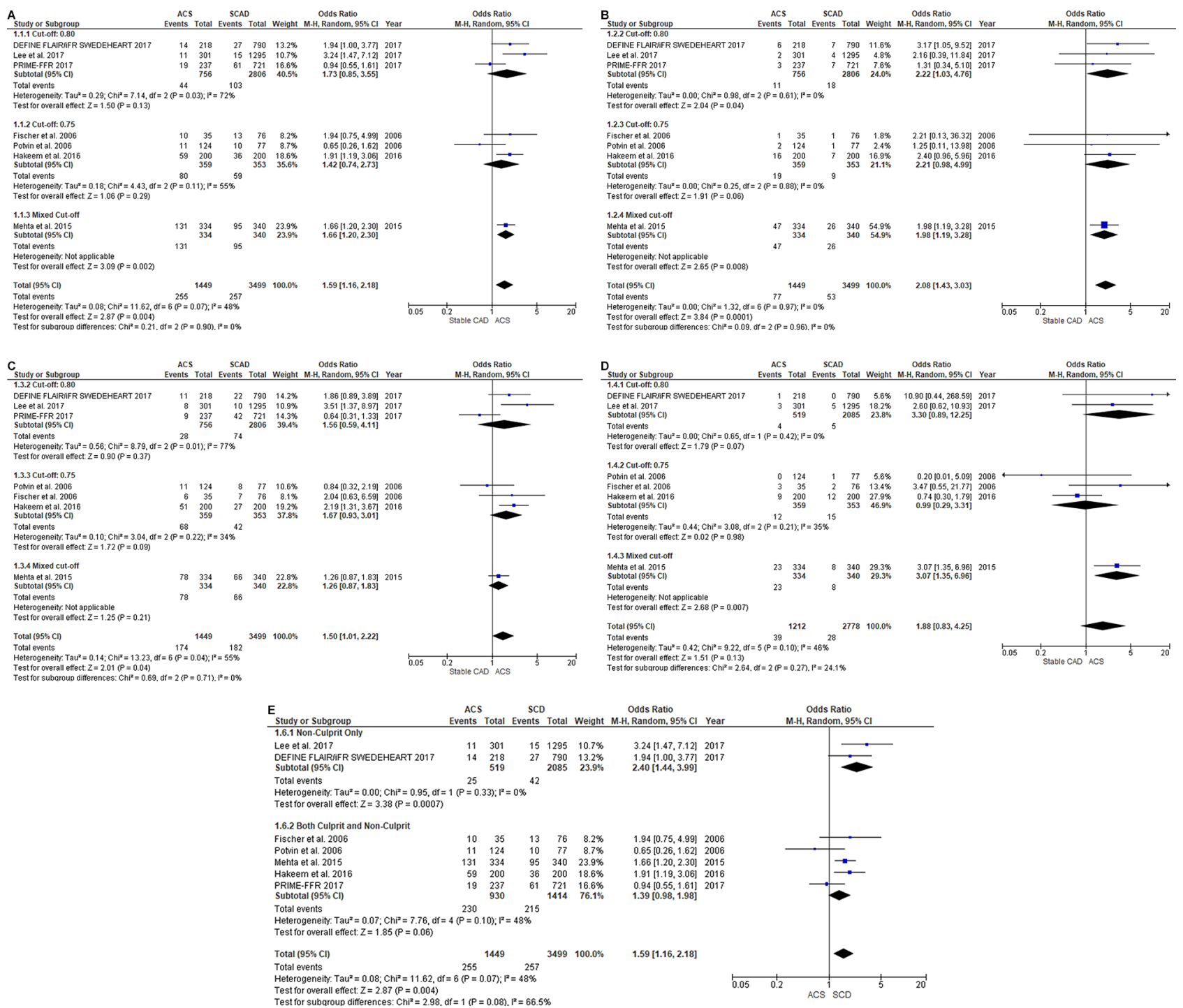

Figure 3 FFR guided deferral of PCl in patients with ACS and stable coronary artery disease (SCAD). (A) mace; (B) recurrent MI; (C) unplanned revascularisation; (D) cardiovascular mortality; (E) non-culprit vessel only versus both culprit and non-culprit vessel. ACS, acute coronary syndrome; FFR, fractional flow reserve; MI, myocardial infarction; PCl, percutaneous coronary intervention.

impaired drug-induced coronary vasodilatation and subsequently the attenuation of the trans-stenotic pressure gradient. ${ }^{23} 25$ This then leads to the underestimation of the lesion severity ${ }^{8}$ and misclassification, particularly if the FFR value is within the diagnostic grey zone. In the setting of ST-elevation myocardial infarction (STEMI), FFR in the culprit arteries following PCI is higher than in patients with SA despite similar intravascular ultrasound parameters, particularly if coronary flow postprocedure was suboptimal. ${ }^{26}$ This is especially marked when the MVD is most apparent. ${ }^{8}$ MVD is also present in non-culprit arteries in patients with NSTEACS, with previous study demonstrating an underestimation of lesion severity by FFR values. ${ }^{27}$

The time required for the microcirculation to normalise is uncertain. One study found that the vasodilatory capacity of the coronary microcirculation in the culprit lesion territory recovered within 4 days of symptom onset in patients with NSTEMI. ${ }^{28}$ Whether FFR measurement should therefore be postponed until such time is unclear, particularly given the importance of early intervention in these patients. It is also not known if the process of microvascular recovery differs between the culprit and non-culprit territories, as the FFR value for the non-culprit lesions appeared to remain stable over time ${ }^{29}$ despite a significantly impaired culprit territory microcirculation in patients with STEMI. ${ }^{28}$ Consequently, deferral of invasive therapy on the basis FFR values obtained acutely, particularly in the presence of large infarction and impaired baseline coronary microcirculation, ${ }^{28}$ remains uncertain and should be treated with caution.

Last, whether the same FFR cut-off values originally derived in a stable population can be applied in those with ACS, particularly following an acute presentation, is unclear and disputed by some. In truth, the relationship between FFR and prognosis is a continuum, and the 
FFR at which PCI achieves a better outcome than medical therapy alone may depend on patient's presentation, baseline attributes and clinical outcomes of interest. ${ }^{30}$

\section{Alternative strategies in lesion assessment}

The discriminatory value of FFR in the setting of ACS may be augmented by other measures. Vulnerable plaques predicts cardiac events and are more prevalent in patients with ACS than stable coronary artery disease. ${ }^{31}$ Plaque characterisation with intracoronary imaging, therefore, may compliment physiological lesion assessment in patients with ACS , particularly when the values are in the grey zone. This combined approach has not been formally validated, however. Further, FFR has been shown to predict plaque vulnerability, which is independent of the extent of luminal stenosis. ${ }^{32-34}$ The added value of intracoronary imaging in this setting therefore requires further examination.

It is also important to ensure the attainment of maximal hyperaemia prior to FFR measurements. To this end, evidence would favour the use of intravenous rather than intracoronary route of adenosine administration, particularly as the former approach also permits FFR pull-back and assessment of more complex lesions. ${ }^{35}$ There appears to be no difference between the use of peripheral and central venous access for adenosine delivery, or with a dose beyond $140 \mu \mathrm{g} / \mathrm{kg} / \mathrm{min}$ in achieving steady state hyperaemia and therefore an informative FFR value. ${ }^{36}$

Alternatively, adenosine-free index of coronary stenosis such as the instantaneous wave-free ratio (iFR) may facilitate case selection for PCI. While a detailed discussion of these indices is beyond the scope of this manuscript, previous studies have demonstrated a strong correlation between iFR and coronary flow and coronary flow reserve. ${ }^{37} 38$ This is of particular significance especially in patients with discrepant FFR and iFR, where iFR may offer added discriminatory value ${ }^{17} 38$ without significantly compromising patient outcome. ${ }^{14}{ }^{15}$ Like FFR however, these indices do have limitations. Operators need to be aware of these and exercise due diligence when performing physiology-guided interventions in patients with ACS. Further data on the optimal treatment approach in this group of patients and new systemic therapies are required to bridge this knowledge and therapeutic gap.

\section{LIMITATION}

Several limitations merit considerations. First, the lack of primary data meant that patient level analyses could not be performed. Second, the observational nature of some of the included studies meant not all biases and confounders could be confidently accounted for by this analysis. Third, most studies failed to report the time interval between patients' symptom onset and their procedures, thus making it difficult to comment on the optimal timing of FFR measurement. Fourth, the utility of FFR in the culprit versus non-culprit lesions in the setting of ACS remains unclear, as most studies did not separate the two in their analyses. We speculate, however, that such distinction may have been difficult in some patients. Finally, the number of studies included in this study was small, which reflects the paucity of evidence in this space. The results of this analysis should therefore be viewed as hypothesis generating, although an appraisal of the existing literature remains a valuable exercise.

\section{CONCLUSION}

In patients with ACS, an FFR-guided treatment strategy and deferral of PCI on the basis of a non-ischaemic FFR $(>0.75-0.80)$ was associated with a worse clinical outcome than patients with SA. The therapeutic benefit of the current approach of FFR-guided multivessel PCI in patients with ACS requires further validation in largescale prospective studies.

Contributors KPL and NEJW were responsible for the original idea. KPL contributed to the planning and conduct of the study as well as data analyses and the drafting of this manuscript. SPH, S-YMO and NEJW provided critical review and contributed to the drafting of this manuscript. KPL is responsible for the overall content of this manuscript as the guarantor.

Funding The authors have not declared a specific grant for this research from any funding agency in the public, commercial or not-for-profit sectors.

Competing interests None declared.

Patient consent Not required.

Provenance and peer review Not commissioned; externally peer reviewed.

Open access This is an open access article distributed in accordance with the Creative Commons Attribution Non Commercial (CC BY-NC 4.0) license, which permits others to distribute, remix, adapt, build upon this work non-commercially, and license their derivative works on different terms, provided the original work is properly cited, appropriate credit is given, any changes made indicated, and the use is non-commercial. See: http://creativecommons.org/licenses/by-nc/4.0

\section{REFERENCES}

1. Tonino PA, De Bruyne B, Pijls NH, et al. Fractional flow reserve versus angiography for guiding percutaneous coronary intervention. N Engl J Med 2009;360:213-24.

2. De Bruyne B, Pijls NH, Kalesan B, et al. Fractional flow reserveguided $\mathrm{PCl}$ versus medical therapy in stable coronary disease. $\mathrm{N}$ Engl J Med 2012;367:991-1001.

3. Engstrøm T, Kelbæk H, Helqvist $\mathrm{S}$, et al. Complete revascularisation versus treatment of the culprit lesion only in patients with STsegment elevation myocardial infarction and multivessel disease (DANAMI-3-PRIMULTI): an open-label, randomised controlled trial. The Lancet 2015;386:665-71.

4. Smits PC, Abdel-Wahab M, Neumann FJ, et al. Fractional flow reserve-guided multivessel angioplasty in myocardial infarction. $N$ Engl J Med 2017;376:1234-44.

5. Layland J, Oldroyd KG, Curzen N, et al. Fractional flow reserve vs. angiography in guiding management to optimize outcomes in non-ST-segment elevation myocardial infarction: the British Heart Foundation FAMOUS-NSTEMI randomized trial. Eur Heart $J$ 2015;36:100-11.

6. Marques KM, Knaapen P, Boellaard R, et al. Hyperaemic microvascular resistance is not increased in viable myocardium after chronic myocardial infarction. Eur Heart J 2007;28:2320-5.

7. Ntalianis A, Sels JW, Davidavicius G, et al. Fractional flow reserve for the assessment of nonculprit coronary artery stenoses in patients with acute myocardial infarction. JACC Cardiovasc Interv 2010;3:1274-81.

8. Cuculi F, De Maria GL, Meier P, et al. Impact of microvascular obstruction on the assessment of coronary flow reserve, index of microcirculatory resistance, and fractional flow reserve after ST-segment elevation myocardial infarction. J Am Coll Cardiol 2014;64:1894-904. 
9. Fischer JJ, Wang XQ, Samady H, et al. Outcome of patients with acute coronary syndromes and moderate coronary lesions undergoing deferral of revascularization based on fractional flow reserve assessment. Catheter Cardiovasc Interv 2006;68:544-8.

10. Potvin JM, Rodés-Cabau J, Bertrand OF, et al. Usefulness of fractional flow reserve measurements to defer revascularization in patients with stable or unstable angina pectoris, non-ST-elevation and ST-elevation acute myocardial infarction, or atypical chest pain. Am J Cardiol 2006;98:289-97.

11. Sels JW, Tonino PA, Siebert U, et al. Fractional flow reserve in unstable angina and non-ST-segment elevation myocardia infarction experience from the FAME (Fractional flow reserve versus Angiography for Multivessel Evaluation) study. JACC CardiovasC Interv 2011;4:1183-9.

12. Masrani Mehta S, Depta JP, Novak E, et al. Association of lower fractional flow reserve values with higher risk of adverse cardiac events for lesions deferred revascularization among patients with acute coronary syndrome. J Am Heart Assoc 2015;4:e002172.

13. Hakeem A, Edupuganti MM, Almomani A, et al. Long-term prognosis of deferred acute coronary syndrome lesions based on nonischemic fractional flow reserve. J Am Coll Cardiol 2016;68:1181-91.

14. Davies JE, Sen S, Dehbi HM, et al. Use of the instantaneous wave-free ratio or fractional flow reserve in PCl. N Engl J Med 2017;376:1824-34.

15. Götberg M, Christiansen EH, Gudmundsdottir IJ, et al. Instantaneous Wave-free Ratio versus Fractional Flow Reserve to Guide PCl. N Engl J Med 2017;376:1813-23.

16. Van Belle E, Baptista SB, Raposo L, et al. Impact of routine fractional flow reserve on management decision and 1-year clinical outcome of patients with acute coronary syndromes: PRIME-FFR (Insights From the POST-IT [Portuguese study on the evaluation of ffr-guided treatment of coronary disease] and R3F [French FFR Registry] integrated multicenter registries - implementation of FFR [Fractional Flow Reserve] in Routine Practice). Circ Cardiovasc Interv 2017; 10:e004296

17. Escaned J, Ryan N, Mejía-Rentería $\mathrm{H}$, et al. Safety of the deferral of coronary revascularization on the basis of instantaneous wave-free ratio and fractional flow reserve measurements in stable coronary artery disease and acute coronary syndromes. JACC CardiovasC Interv 2018;11:1437-49.

18. Lee JM, Choi KH, Koo BK, et al. Prognosis of deferred non-culprit lesions according to fractional flow reserve in patients with acute coronary syndrome. Eurolntervention 2017;13:e1112-e1119.

19. Rioufol G, Finet G, Ginon I, et al. Multiple atherosclerotic plaque rupture in acute coronary syndrome: a three-vessel intravascular ultrasound study. Circulation 2002;106:804-8.

20. Buffon A, Biasucci LM, Liuzzo G, et al. Widespread coronary inflammation in unstable angina. N Engl J Med 2002;347:5-12.

21. Wald DS, Morris JK, Wald NJ, et al. Randomized trial of preventive angioplasty in myocardial infarction. N Engl J Med 2013;369:1115-23.

22. Gershlick AH, Khan JN, Kelly DJ, et al. Randomized trial of complete versus lesion-only revascularization in patients undergoing primary percutaneous coronary intervention for STEMI and multivessel disease: the CvLPRIT trial. J Am Coll Cardiol 2015;65:963-72.

23. Lee JM, Kim HK, Lim KS, et al. Influence of local myocardial damage on index of microcirculatory resistance and fractional flow reserve in target and nontarget vascular territories in a porcine microvascular injury model. JACC Cardiovasc Interv 2018;11:717-24.

24. Roe MT, Ohman EM, Maas AC, et al. Shifting the open-artery hypothesis downstream: the quest for optimal reperfusion. J Am Coll Cardiol 2001;37:9-18.
25. van de Hoef TP, Nolte F, Echavarrla-Pinto M, et al. Impact of hyperaemic microvascular resistance on fractional flow reserve measurements in patients with stable coronary artery disease: insights from combined stenosis and microvascular resistance assessment. Heart 2014;100:951-9.

26. Tamita K, Akasaka T, Takagi T, et al. Effects of microvascular dysfunction on myocardial fractional flow reserve after percutaneous coronary intervention in patients with acute myocardial infarction. Catheter Cardiovasc Interv 2002;57:452-9.

27. Niccoli G, Falcioni E, Cosentino N, et al. Impact of accuracy of fractional flow reserve to reduction of microvascular resistance after intracoronary adenosine in patients with angina pectoris or non-ST-segment elevation myocardial infarction. Am J Cardiol 2014;113:1461-7.

28. Layland J, Carrick D, McEntegart M, et al. Vasodilatory capacity of the coronary microcirculation is preserved in selected patients with non-ST-segment-elevation myocardial infarction. Circ Cardiovasc Interv 2013;6:231-6.

29. Musto C, De Felice F, Rigattieri S, et al. Instantaneous wave-free ratio and fractional flow reserve for the assessment of nonculprit lesions during the index procedure in patients with ST-segment elevation myocardial infarction: the WAVE study. Am Heart $J$ 2017; 193:63-9.

30. Johnson NP, Tóth GG, Lai D, et al. Prognostic value of fractional flow reserve: linking physiologic severity to clinical outcomes. J Am Coll Cardiol 2014;64:1641-54

31. Ando H, Amano T, Matsubara T, et al. Comparison of tissue characteristics between acute coronary syndrome and stable angina pectoris. Circulation Journal 2011;75:383-90.

32. Gaur S, Øvrehus KA, Dey D, et al. Coronary plaque quantification and fractional flow reserve by coronary computed tomography angiography identify ischaemia-causing lesions. Eur Heart $J$ 2016;37:1220-7.

33. Park HB, Heo R, ó Hartaigh B, et al. Atherosclerotic plaque characteristics by $\mathrm{CT}$ angiography identify coronary lesions that cause ischemia: a direct comparison to fractional flow reserve. JACC Cardiovasc Imaging 2015;8:1-10.

34. Tanaka S, Noda T, Segawa T, et al. Relation between functional stenosis and tissue characterization of intermediate coronary plaques in patients with stable coronary heart disease. J Cardiol 2010;55:296-302.

35. Layland J, Carrick D, Lee M, et al. Adenosine: physiology, pharmacology, and clinical applications. JACC Cardiovasc Interv 2014;7:581-91.

36. De Bruyne B, Pijls NH, Barbato E, et al. Intracoronary and intravenous adenosine 5 '-triphosphate, adenosine, papaverine, and contrast medium to assess fractional flow reserve in humans. Circulation 2003;107:1877-83

37. Petraco R, van de Hoef TP, Nijier S, et al. Baseline instantaneous wave-free ratio as a pressure-only estimation of underlying coronary flow reserve: results of the JUSTIFY-CFR Study (Joined coronary pressure and flow analysis to determine diagnostic characteristics of basal and hyperemic indices of functional lesion severity-coronary flow reserve). Circ Cardiovasc Interv 2014;7:492-502.

38. Cook CM, Jeremias A, Petraco R, et al. Fractional flow reserve/ instantaneous wave-free ratio discordance in angiographically intermediate coronary stenoses: an analysis using dopplerderived coronary flow measurements. JACC Cardiovasc Interv 2017;10:2514-24. 